\title{
1 A single ectomycorrhizal plant root system includes a 2 diverse and spatially structured fungal community
}

4 Ella Thoen $^{1 *}$, Anders B. Aas ${ }^{1,2}$, Unni Vik ${ }^{1}$, Anne K. Brysting ${ }^{1}$, Inger Skrede ${ }^{1}$, Tor Carlsen ${ }^{3}$, $5 \quad$ Håvard Kauserud ${ }^{1}$

$6 \quad{ }^{1}$ Section for Genetics and evolutionary biology (EVOGENE), Department of Biosciences, University of

7 Oslo, PO box 1066 Blindern, NO-0316 Oslo, Norway. ${ }^{2}$ Bymiljøetaten Oslo Kommune PO box 636 Løren,

80507 Oslo, Norway. ${ }^{3}$ The Natural History museum, University of Oslo, PO box 1172 Blindern, 0318

9 Oslo, Norway

10 *Corresponding author: Ella Thoen, ella.thoen@ibv.uio.no, +47 98878755,

11 https://orcid.org/0000-0001-6169-0541, Department of Biosciences, University of Oslo, PO box 121066 Blindern, 0316 Oslo, Norway. 


\section{Abstract}

16 Although only a relatively small proportion of plant species form ectomycorrhizae with fungi, it

17 is crucial for growth and survival for a number of widespread woody plant species. Few studies

18 have attempted to investigate the fine scale spatial structure of entire root systems of adult

19 ectomycorrhizal (EcM) plants. Here, we use the herbaceous perennial Bistorta vivipara to map

20 the entire root system of an adult EcM plant and investigate the spatial structure of its root

21 associated fungi. All EcM root tips were sampled, mapped and identified using a direct PCR

22 approach and Sanger sequencing of the ITS region. A total of $32.1 \%$ of all sampled root tips

23 (739 of 2302) were successfully sequenced and clustered into 41 OTUs. We observed a clear

24 spatial structuring of the root associated fungi within the root system. Clusters of individual

25 OTUs were observed in the younger parts of the root system, consistent with observations of

26 priority effect in previous studies, but were absent from the older parts of the root system. This

27 may suggest a succession and fragmentation of the root-associated fungi even at a very fine

28 scale, where competition likely comes into play at different successional stages within the root 29 system.

30

31 Keywords: ectomycorrhiza, Bistorta vivipara, fine-scale spatial structure, priority effects 


\section{Introduction}

33 Although a relatively small number of plant species form ectomycorrhizae compared to

34 arbuscular mycorrhiza (Brundrett and Tedersoo 2018), ectomycorrhizal (EcM) fungi is crucial

35 for growth and survival of a number of ecologically and economically important woody plant

36 species in boreal, temperate and arctic regions (Smith and Read 2008). In these environments,

37 EcM fungi have been shown to play a prominent role including, but not limited to; carbon

38 sequestration (Clemmensen et al. 2013; Averill et al. 2014), nutrient cycling and decomposition

39 of organic matter (Lindahl et al. 2007; Kyaschenko et al. 2017; Sterkenburg et al. 2018).

40 An increasing amount of studies have addressed the spatial variation in EcM fungal

41 communities, ranging from local (Genney et al. 2006; Buée et al. 2007; Bahram et al. 2011,

42 2015; Anderson et al. 2014), regional (Jarvis et al. 2013; Miyamoto et al. 2015), continental

43 (Talbot et al. 2014; Glassman et al. 2015; van der Linde et al. 2018) and even global scales

44 (Tedersoo et al. 2010; Brundrett and Tedersoo 2018). At all scales, there is a high level of

45 patchiness and a rapid turnover in species composition. Fungal communities in forest ecosystems

46 are auto-correlated at relatively small spatial scales, typically less than $3 \mathrm{~m}$ (Lilleskov et al.

47 2004; Pickles et al. 2012), and forest microsite affects the EcM community composition

48 (Tedersoo et al. 2008). At smaller scales, the abundance of EcM fungi in a mixed forest is highly

49 variable at a $5 \mathrm{~cm}$ scale, and a complete turnover in species composition can be observed at a 50

50 cm scale (Tedersoo et al. 2003).

51 Although the community composition of EcM fungi has been widely studied at relatively local

52 scales $(\mathrm{cm}-\mathrm{m})$, less is known about the spatial distribution at smaller scales $(\mu \mathrm{m}-\mathrm{cm})$ and within

53 single root systems (Bogar and Peay 2017). Although individual EcM genets can cover relatively

54 large belowground areas, (up to $18 \mathrm{~m}$ in some species) (Dahlberg and Stenlid 1994; Bergemann

55 and Miller 2002), EcM root tips may be located less than $1 \mathrm{~mm}$ from each other in the soil, and

56 individual hyphae interact at $\mu \mathrm{m}$ scale (Bogar and Peay 2017). The few studies conducted on a

57 'single host plant scale` show that the EcM fungal community is very diverse even within an

58 individual host plant. A single tree of Populus tremula hosted more than 200 root associated

59 fungal species, as well as dozens of individuals of the same species (Bahram et al. 2011). Even in

60 seedlings and herbaceous plants, the number of species is surprisingly high. The number of root

61 associated operational taxonomic units (OTUs, a proxy for species) may range from 37 to 159 
within single root systems of the EcM forming plant B. vivipara (Yao et al. 2013), and Yoshida

63 et al. (2014) found 40 species of EcM fungi associated with three Tsuga diversifolia seedlings.

64 The processes structuring EcM fungal communities and maintaining species richness at finer

65 scales in root systems may be attributed to several selective processes, ecological drift and

66 dispersal limitations (Bogar and Peay 2017). EcM fungi are phylogenetically diverse, and their

67 morphology when colonizing root tips varies between species, from contact exploration types

68 (i.e. no emanating hyphae from the mantle covering the plant root tip), to rhizomorphic, long-

69 distance exploration types (Agerer 2001). However, exploration type is often relatively

70 conserved within genera or even family (Agerer 2001). The exploration types of EcM fungi

71 associated with plant roots have been shown to correlate with both root density (Peay et al. 2011)

72 and forest age (Clemmensen et al. 2015). Therefore, exploration types may potentially be

73 important in structuring communities also at finer scales. Stochastic events, such as timing of

74 arrival of a species, can have major effects on the outcome of the species distribution within a

75 single root system (Kennedy and Bruns 2005; Kennedy et al. 2009), and may, at least in part,

76 explain some variation in root associated communities found across host plants at small scales

77 (Yao et al. 2013). A stochastic event, such as the timing of arrival of a species (i.e. priority

78 effect) is thought to play a major role in structuring EcM communities. There are, however, some

79 contrasting results regarding the importance of priority effects in EcM fungal communities.

80 Lilleskov \& Bruns (2003) deemed competition more important than the priority effect, as a

81 slower colonising species had a greater competitional advantage, in spite of a later colonisation.

82 On the contrary, Kennedy \& Bruns (2005) found competition to be an important factor, but a

83 strong priority effect determined the outcome of the dominant species in their EcM root systems.

84 They later confirmed that root systems were dominated by the earlier coloniser (Kennedy et al.

85 2009), and that priority effects determined the outcome. Yoshida et al (2014) investigated the

86 fine scale structure of three seedlings of $T$. diversifolia from a natural environment, and similarly

87 observed that the priority effect was likely important in structuring the EcM fungal community,

88 as clusters of root tips colonised by individual species were observed in the root system.

89 However, in mature forest systems, competitive interactions seem to be the determining process

90 in structuring EcM communities at finer scales (Pickles et al. 2012). There is strong evidence

91 that priority effects play important roles in structuring EcM communities for seedlings, however, 
92 EcM competitive patterns may be life-stage dependent (Kennedy et al. 2011), and should thus be 93 investigated further.

94 Most of the evidence for priority effects in EcM fungal communities comes from studies on 95 seedlings. To the best of our knowledge, no exhaustive study looking at fine scale spatial 96 structure of root-associated fungi have been conducted on adult EcM plants (i.e. investigating all 97 EcM root tips within the root system). This may be because most ectomycorrhizae-forming 98 plants are woody plant species with large root systems, and thus more challenging to investigate 99 in detail. However, a few herbaceous plants also form ectomycorrhizae (Smith and Read 2008), 100 including the Alpine bistort; a small, perennial herb (Bistorta vivipara (L.) Delarbre, synonym

101 Persicaria vivipara (L). Ronse Decr.). Because of its small, condensed root system, it is possible 102 to investigate the entire root systems, and several studies have shown that B. vivipara hosts 103 phylogenetically diverse root associated fungal communities (Brevik et al. 2010; Blaalid et al. 104 2012, 2014; Kauserud et al. 2012; Yao et al. 2013; Botnen et al. 2014; Mundra et al. 2015b).

105 In the present study, we take advantage of the small root system of $B$. vivipara to assess the 106 diversity and spatial distribution of the root associated fungal community within a single plant 107 root system at a root-tip level. The root morphology of B. vivipara also allows us to investigate 108 root system age, as the plant grows directionally with an unbranched rhizome, where the growth 109 of the rhizome is restricted to the distal end (Diggle 1997). Numerous fine roots, where the EcM 110 tips are formed, grows out from the rhizome. The exact turnover rates of B. vivipara fine roots 111 are not known. However, in a study from the arctic focusing on temporal changes in the root112 associated fungal communities of B. vivipara (Mundra et al. 2015a), fine roots were observed 113 year-round, indicating they have a longer persistence time, not just being produced every 114 growing season. Thus, we can expect that the fine roots in general are younger in the actively 115 growing end of the rhizome and that there is an age gradient within the root system.

116 Due to the likely influence of priority effects, we hypothesise that there will be a spatial 117 clustering of fungal species in the root system, as observed earlier in other plant root systems 118 (Kennedy and Bruns 2005; Kennedy et al. 2009; Yoshida et al. 2014). If competition and 119 succession is a stronger driver of structuring the fungal community, we expect to observe a 120 weaker signal of priority effects, and less spatial clustering of species. This may also be reflected 121 as a change in exploration types along the age gradient. To investigate this, a single EcM root 
122 system of B. vivipara was dissected and all EcM root tips mapped and sampled. A direct PCR 123 approach, where the entire ITS region was amplified from crushed root tips without DNA

124 extraction, followed by Sanger sequencing, was used to identify fungal OTUs within the root 125 system of one B. vivipara individual. 


\section{Materials \& Methods}

\section{Host plant description}

128 The host plant, B. vivipara, is a long-lived perennial in the family Polygonaceae, first reported as

129 ectomycorrhizae-forming by Hesselman (1900) and later confirmed in several studies (e.g.

130 Massicotte et al. 1998; Brevik et al. 2010; Kauserud et al. 2012; Botnen et al. 2014). The plant is

131 widely distributed in the Northern Hemisphere and is common in arctic and alpine ecosystems

132 (Jonsell 2000). Reproductive structures include flowers, but more commonly reproduction is

133 through asexual bulbils. The plant produces an unbranched rhizome (see Fig. 1) that grows

134 plagiotropically 3-4 cm below ground, from which fine roots are growing (Diggle 1997). The

135 fungal community of $B$. vivipara has been found to be highly diverse, comprising a wide range

136 of both endo- and ectomycorrhizal fungi as well as saprotrophs, parasites and endophytes.

137 Basidiomycota and Ascomycota have been found to be the most dominant phyla, although

138 Glomeromycota and Zygomycota are found as well (Massicotte et al. 1998; Carlsen 2002;

139 Eriksen et al. 2002; Brevik et al. 2010; Blaalid et al. 2012, 2014; Yao et al. 2013; Botnen et al.

140 2014).

141

142 Field description and sample preparation

143 Sampling was conducted August 1, 2012 at the glacier foreland in front of Blåisen, Finse,

144 Norway $\left(60^{\circ} 33^{\prime} \mathrm{N}, 7^{\circ} 31^{\prime} \mathrm{E}, 1388 \mathrm{~m}\right.$ a.s.1.), which is an alpine ecosystem. The sampling site was

145 sparsely vegetated, with Salix herbaceae L. and B. vivipara as the dominating plant species. The

146 site was chosen because of the very sparse vegetation, making it possible to sample and analyse a

147 single root system without breaking off roots due to entanglement and intermixing with roots

148 from other plants.

149 One randomly chosen B. vivipara plant and its entire root system was dug up and stored in a zip-

150 lock bag in a cooler for transportation, and later in a fridge at $4{ }^{\circ} \mathrm{C}$ until further processing. The

151 root system was rinsed one day after sampling and transportation, first gently in tap water and

152 then thoroughly in distilled water. A dissecting microscope was used to inspect the roots to

153 ensure that all debris and soil attached to the plant's rhizome or roots were removed. As a final

154 rinsing step, the roots were washed three times in milli- $\mathrm{Q} \mathrm{H}_{2} \mathrm{O}\left(\mathrm{mqH}_{2} \mathrm{O}\right)$ for $30 \mathrm{sec}$, before 
excess water was gently dried off with sterile tissue paper. The root system was stored in a petri

156 dish at $4{ }^{\circ} \mathrm{C}$ until further processing.

157

158

159

160

161

162

163

164

165

166

167

168

169

170

171

172

173

174

175

176

177

178

179

180

181

182

183

184

\section{Root tip sampling}

Within two days after sampling, the rhizome along with attached fine root was dissected into four approximately equal sized parts, which were named R1, R2, R3 and R4 (Fig. 1). R1 was the distal end (i.e. where the current year's leaves and flowers grow) and thereby the youngest part of the rhizome and R4 the proximal and oldest part of the rhizome (Diggle 1997). The number of fine roots present at each rhizome part was highly variable, ranging from three (R2, R4) to 13 (R1), where newly formed roots were only observed in R1. The fine roots were numbered chronological from distal to proximal end and cut off from the rhizome, totalling 29 fine roots from the entire root system, and further, stored in individual petri dishes at $-18{ }^{\circ} \mathrm{C}$ until further processing. Root tips were sampled and numbered from top (closest to the rhizome) to bottom of the fine roots and simultaneously mapped onto an overview photograph to keep track of their relative position in the root system (see also Fig. S2 for a photograph of a fine root). If root tips were located on branches from the main fine root, this was noted as well. The number of root tips per fine root varied from three to 278 and were relatively even spaced on the fine roots. All root tips had either a fungal mantle or signs of fungal colonisation (i.e. hyphae were present when observed under the dissection microscope).

Dissection of the fine roots was conducted in a petri dish with $\mathrm{mqH}_{2} \mathrm{O}$ under a dissecting microscope. Each root tip was cut off using a scalpel, transferred to an Eppendorf tube with 100 $\mu \mathrm{mqH} \mathrm{H}_{2} \mathrm{O}$ and stored at $-18{ }^{\circ} \mathrm{C}$. The total number of root tips in the root system was 2302 .

\section{Molecular analyses}

The ITS region was used to barcode the fungal component of the root tips. The fungal-specific primers ITS1F and ITS4, targeting the entire ITS region including ITS1, 5.8S and ITS2 (White et al. 1990; Gardes and Bruns 1993), were used for all PCR reactions. Before PCR, all samples were crushed using two Qiagen ${ }^{\circledR}$ Tungsten Carbide beads $(3 \mathrm{~mm}$ ) on a Rech Ball Mill MM301 (Verder Scientific, Haan, Germany) for 2 x 2 min at 20 rounds per sec. Several approaches were used to amplify as many root tips as possible. The Thermo Scientific Direct PCR Plant kit (Thermo Fisher Scientific Inc., Waltham, Massachusetts, USA) was used for DNA amplification of most samples. The kit allows for direct amplification without time-consuming DNA extraction 
and purifications steps. The kit was modified for fungi as follows: For each reaction $2 \mu 1$ crushed root tip in water (DNA template) was added to a master mix consisting of $10 \mu 13 \mathrm{X}$ Plant buffer, $2 \mu 15 \mu \mathrm{M}$ ITS1F, $2 \mu 15 \mu \mathrm{M}$ ITS4, $3.6 \mu 1 \mathrm{mqH}_{2} \mathrm{O}$ and $0.4 \mu \mathrm{l}$ Phire HotStart Polymerase (Thermo Fisher Scientific Inc., Waltham, Massachusetts, USA). The PCR reaction was run with an initial step for $5 \mathrm{~min}$ at $98{ }^{\circ} \mathrm{C}, 40$ cycles of denaturation at $98{ }^{\circ} \mathrm{C}$ for $20 \mathrm{sec}$, primer binding at $53{ }^{\circ} \mathrm{C}$ for $20 \mathrm{sec}$ and extension at $72{ }^{\circ} \mathrm{C}$ for $2 \mathrm{~min}$, and a final extension at $72{ }^{\circ} \mathrm{C}$ for $2 \mathrm{~min}$, before cooling down to $10{ }^{\circ} \mathrm{C}$. For samples with unsuccessful amplification after the first setup, a new PCR reaction was run as previously, but the number of PCR cycles was reduced to 36 . For those that still did not amplify, a $50 \mathrm{x}$ dilution of the crushed root tips was used as template, using the above temperatures and 36 cycles. Further, as a last try, the REDExtract-N-Amp Plant PCR Kits (Sigma), slightly modifying the manufacturer's instructions, was used to amplify the root tips that had not worked in the initial amplification attempts. In short, $2 \mu 1$ of the crushed root tip and $\mathrm{mqH}_{2} \mathrm{O}$ solution was incubated in Extraction solution for $10 \mathrm{~min}$ at $95{ }^{\circ} \mathrm{C}$ before adding $30 \mu \mathrm{l}$ of Dilution buffer. PCR was set up using illustra ${ }^{\mathrm{TM}}$ PuReTaq Ready-To-Go ${ }^{\mathrm{TM}}$ PCR Beads, according to the manufacturer's instructions. The PCR products were cleaned using illustra ${ }^{\mathrm{TM}}$ $\operatorname{ExoStar}^{\mathrm{TM}}$ (VWR international Ldt., Dublin, Ireland) and submitted for Sanger sequencing at the ABI lab, University of Oslo or to the GATC biotech (Konstanz, Germany). The ITS amplicons were sequenced in one direction only. Initially, ITS4 was used as a sequencing primer for $63.9 \%$ of the amplicons, but due to poor quality of some of the sequences, ITS5 was used for the remaining samples.

\section{Bioinformatics and statistical analyses}

A total of 1891 ITS sequences were obtained and manually trimmed and edited using Geneious® 8.1.7 Biomatters (Biomatters Ltd, Auckland). After removing sequences with low quality chromatograms, i.e. sequences with more than ten ambiguities and read-length shorter than 350 base pairs (bp), 1080 sequences remained for further analyses.

Because several of the PCR negatives produced clear bands on the gel, additional quality control of the sequences was necessary to avoid including contaminants in the dataset. All 1080 sequences were aligned using custom settings in the MAFT package included in Geneious ${ }^{\circledR}$ 8.1.7. A neighbour joining (NJ) tree was constructed from the alignment using custom settings in Genious ${ }^{\circledR}$ 8.1.7. Clusters were manually extracted from the cladogram. Each cluster of 
216 sequences was re-aligned using MAFT (as above), further manually inspected, edited and quality

217 checked within the alignment and one sequence from each alignment of identical sequences was

218 BLASTED against UNITE (Kõljalg et al. 2013). Clusters of sequences that included negative

219 controls were altogether removed from the dataset. Further, sequences that had top hits to known

220 laboratory contaminants and sequences from several ubiquitous polyporid fungi were removed

221 from the dataset (Table S2). Only sequences with top hit to EcM fungi and known root

222 associated fungi were included, with a few exceptions (see discussion).

223 The remaining dataset of 739 sequences were clustered into OTUs using BlastClust (Alva et al.

224 2016) with $97.0 \%$ sequence similarity and minimum $70.0 \%$ query cover. All OTUs were

225 manually inspected in Geneious ${ }^{\circledR}$ 8.1.7. Most of the OTUs contained only identical sequences.

226 All further analyses were conducted in R (R Core Team 2017). To examine if the sampling depth

227 was sufficient, species accumulation curves were constructed (Oksanen et al. 2013), with fine

228 roots as sites. Richness and Shannon diversity index (H') was calculated for each part of the

229 rhizome and each fine root using the vegan package (Oksanen et al. 2013) to assess species

230 richness and diversity. OTUs that had taxomonic affinity to $\mathrm{EcM}$ fungi were assigned to

231 exploration types by species using DEEMY (Agerer \& Rambold, 2004-2018). For instances

232 where OTUs had poor match to a species, but exploration type was consistent throughout the

233 genus, exploration type was also assigned. Eleven OTUs were assigned to mycorrhizal

234 exploration type; contact, short distance smooth, medium distance smooth or medium distance

235 fringe according to DEEMY.

237 Spatial structure

238 To investigate spatial structure in the root system, a matrix of the abundances of OTUs per fine 239 root (site) was constructed. Fine roots with less than ten observations (successfully sequenced 240 root tips) were excluded, leaving 18 fine roots and 701 root tips. Because ordination results 241 strongly depend on the choice of ordination method and weighting function, a multiple parallel 242 ordination (MPO) approach was used, as recommended by van Son \& Halvorsen (2014). MPO 243 was conducted using fine roots as plots, and OTUs as "species" observations. Three different 244 weighting functions (data transformations) using power-function weighting (van der Maabel 245 1979; Clymo 1980); with raw data $(w=1)$, intermediate weighting $(w=0.5)$ and presence-absence $246(w=0)$ were used, as described in van Son \& Halvorsen (2014, for details see also Økland 1986). 
247 If ordination structure is similar between parametric and non-parametric ordination methods, 248 there is indication of a robust structuring of the dataset (Son and Halvorsen 2014). Therefore two 249 ordination methods were used: Detrended correspondence analyses (DCA, Hill \& Gauch 1980) 250 and global non-metric multidimensional scaling (GNMDS, Kruskal 1964) ordinations were 251 constructed using the vegan package v. 2.0-10 (Oksanen et al. 2013). For the DCA ordinations, 252 default settings were used. For GNMDS the following settings were used: two dimensions, 253 dissimilarity measure used was Jaccard distance with an isometric geodesic step-across measure 254 for Jaccard distance $>0.8$ (Tenenbaum 2000), maximum number of iterations was set to 200 and 255100 random initial starting configurations were used. The same settings were used for all three 256 weighting functions. To see if axes of GNMDS and DCA correlated and which variables 257 covaried with each other and the ordination axes, a correlation test with Kendall's $\tau$ rank 258 correlation coefficient (Kendall 1938; Kruskal 1958) was conducted. In addition, exploration 259 types, diversity (Shannon's H), richness per fine root, number of root tips (i.e. root density), 260 length, branching and position of fine roots along the rhizome were fitted onto the ordination 261 diagram using the envar function in the vegan package v. 2.0-10 (Oksanen et al. 2013), where 262 only variables with a significant correlation to ordination axes were plotted.

264 To investigate whether a species tended to form clusters within the root system (i.e. indication of 265 priority effect), a Jaccard distance matrix using the assigned OTU per root tip was constructed, 266 where dissimilar OTUs would get a value of 1 and identical OTUs would get a value of 0 . We 267 used an approximation, because it is impossible to accurately estimate the actual position of root 268 tips and how the fine roots are oriented in the soil before rinsing. Even though the spatial 269 orientation in the soil and the actual physical distance between each root tip are unknown, root 270 tips located on the same fine root or branch on fine roots were assumed to be in closer proximity 271 than root tips located on other fine roots and similarly, root tips that were sampled in sequence 272 on the same branch were assumed to be located in close proximity in the soil (see also Fig. 1 for 273 a schematic drawing of the B. vivipara root system and Fig. S2 for a photograph and description 274 of our assumptions). Accordingly, three distance classes were defined: neighbours (strict 275 neighbouring root tips, sampled in sequence on the same branch), adjacent (root tips sample on 276 the same branch on a fine root) and non-neighbours. These classes were used for constructing a 277 distance matrix, such that strict neighbouring root tips were assigned a spatial distance of 1 , 
278 adjacent root tips were scored as 2-23 (max number of root tips on branches) and non-

279 neighbouring root tips were assigned a much higher number to emphasize non-adjacency

280 (>>300). Because initial tests showed very similar results for neighbouring and adjacent root

281 tips, we only focus on neighbour (i.e. adjacent was merged into the neighbour class) and non-

282 neighbour in the downstream results and discussion. Neighbours and non-neighbours were

283 extracted from the matrix into data frames consisting of values of dissimilarities, so that pairs of

284 root tips sharing an OTU obtained a value of 0 and pairs not sharing an OTU obtained a value of

285 1. The subsets were compared using a two-sided Welch T-test, which takes into account the

286 differences in sample size in the two classes. The root system was analysed as a whole, and

287 subsequently each rhizome part (R1-R4) was analysed separately, to see whether patterns were

288 similar across the entire root system.

289 A Pearson's $\chi^{2}$-test was conducted using the stats package (R Core Team 2017) to see whether

290 the fungal community within parts of the root system was different from other parts than

291 expected by chance. For each OTU, the actual distribution was tested against an expected

292 random distribution in each part of the rhizome. 


\section{Results}

294 After discarding sequences of low quality and tentative contaminants, 739 sequences

295 representing $32.1 \%$ of the total number of root tips in the root system remained for further

296 analyses. The number of fine roots, root tips and OTUs varied considerably throughout the root

297 system, as shown in Table 1. Three fine roots had the appearance of being newly formed (i.e.

298 within the same year), and all three were found attached to R1 of the rhizome. A total of 41

299 fungal OTUs were observed in the root system. A large proportion (60.5\%) of the successfully

300 sequenced root tips was located in the youngest part (R1) of the root system, likely because this

301 was also the part with the largest proportion of fine roots and total root tips. The most

302 successfully sequenced part of the root system was R2, where $53.1 \%$ of all root tips gave an

303 acceptable sequence. The frequency distribution of OTUs showed that the root system was

304 dominated by a few abundant OTUs and $36.6 \%$ of the OTUs was observed once (Fig. 2a). All

305 non-singleton OTUs are listed in Table 2 (singleton OTUs in Table S2). Representative

306 sequences of all OTUs are deposited to GenBank, under accession number MK614825-

307 MK614865.

309 The accumulation curve of OTUs did not level off, which was to be expected since only $32.1 \%$

310 of the root tips in the root system was analysed (Fig. 2b). The fungal diversity, assessed by

311 Shannon's H, varied consistently throughout the root system, with the older parts of the root

312 system (R3 and R4) having higher scores than the younger parts (R1 and R2, Table 1).

313 The majority of OTUs of the sequenced root tips belonged to Basidiomycota (85.3\%) while 13.9

$314 \%$ belonged to Ascomycota. Most of the sequenced root tips (84.6\%) that were included in the

315 analyses had taxonomic affinity to known EcM fungi. More than half $(58.0 \%)$ of the sequenced

316 root tips belonged to the family Thelephoraceae, and the second most common family was

317 Cortinariaceae (10.3\%), followed by Russulaceae (6.2\%). OTU1 (Pseudotomentella sp.) was

318 the most abundant OTU throughout the root system, and more common in the younger part (R1)

319 of the root system than would be expected by chance (Pearson's $\chi^{2}$-test, $\chi 2=40.825$, $\mathrm{df}=3$,

$320 \mathrm{p}<0.001$ ). OTU3 (Cortinarius sp.1) was more abundant in R3 and R4 than expected by chance

321 (Pearson's $\chi^{2}$-test, $\chi^{2}=25.244, \mathrm{df}=3, \mathrm{p}<0.001$ ), and OTU4 (Russula subrubens) was more

322 widespread in the oldest part (R4) than in an expected distribution (Pearson's $\chi^{2}$-test, $\chi^{2}=25.244$,

$323 \mathrm{df}=3, \mathrm{p}<0.001)$. OTU12, although only represented by 10 successfully sequenced root tips, had a 
324 top hit to the Geoglossaceae, Sarcoleotia globosa (99.9\% similarity), which is not a known root 325 associate, but still included in downstream analysis (see discussion). This OTU was only found in the older parts of the root system ( $\mathrm{R} 3$ and $\mathrm{R} 4$, Pearson's $\chi^{2}$-test, $\chi^{2}=14.665, \mathrm{df}=3, \mathrm{p}=0.002$ ).

327 To investigate the spatial structure and compositional variation of fungi in the root system, 328 ordination diagrams of OTU/fine root matrices were constructed, where each sample represents one fine root (as sites). DCA and GNMDS axes were strongly correlated with Kendall's $\tau$ rank correlation test for all ordination methods, and in all ordination diagrams point configurations were similar (Fig. S1), suggesting a robustly structured dataset (Son and Halvorsen 2014). Hence, we only focus on the GNMDS constructed using power-weighting ( $w=0.5)$, which was the

333 GNMDS with the lowest stress values (stress=0.122). The ordination diagram showed a clear 334 spatial structuring of the root-associated fungal community throughout the root system (Fig. 3), 335 where fine roots from the different age classes clustered together. GNMDS1 represented to a 336 large extent the length of the rhizome, as shown by the high correlation of position of fine roots 337 along the rhizome with axis 1 (Fig. 3, Table S3). Fine roots from R1 were located at one end of 338 the axis, and a shift from this part throughout to R4 was observed. Significant variables fitted 339 onto the ordination diagrams showed that diversity was strongly positively correlated with the 340 position of fine roots along the rhizome (proxy for age) and GNMDS1, short smooth exploration 341 type (sh_smooth) was negatively correlated with GNMDS1. This indicate that this exploration 342 type was more common in the younger part of the root system, whilst contact exploration type 343 (contact) was positively correlated with position, indicating that this was more common in the 344 older parts of the root system. The structure along GNMDS2 was mainly driven by richness.

To see whether root tips of the same OTU tended to cluster in the root system, a Bray-Curtis

347 dissimilarity matrix of all successfully sequenced root tips and a distance matrix of their relative 348 position in the root system were used to compare differences in mean dissimilarity between 349 neighboring and non-neighboring root tips using Welch two-sided T-test. The differences 350 between dissimilarities in neighboring and non-neighboring root tips were significant (Welch 351 two-sided T-test, $\mathrm{t}=-12.920, \mathrm{df}=515.620, \mathrm{p}<0.001)$ when analysing root system as a whole. The 352 mean value of adjacent root tips was estimated to 0.53 , meaning that almost half of the 353 neighboring root tips were identified to the same OTU, while for non-neighbors the mean 354 dissimilarity was estimated to 0.82 , meaning that most pairs of root tips throughout the root 
355 system were dissimilar. The different parts of the root system were subjected to the same test, to 356 see whether this pattern varied throughout the root system. The parts R1 and R3 had a significant 357 different mean of dissimilarities in neighboring and non-neighboring (Welch two-sided T-test, $358 \mathrm{t}=11.569, \mathrm{df}=329.900, \mathrm{p}<0.001$, and $\mathrm{t}=4.759, \mathrm{df}=135.590, \mathrm{p}<0.001$, respectively), while in $\mathrm{R} 2$ 359 and R4 the difference was not significant (Welch two-sided T-test, $t=1.727, \mathrm{df}=9.289, \mathrm{p}=0.117$ 360 and $\mathrm{t}=1.213, \mathrm{df}=39.843, \mathrm{p}=0.232$, respectively). There were probably not enough observations in 361 R2 to infer any statistical significance (only 17 successfully sequenced root tips). From R1 362 through R4 there was an increase in estimated mean values for neighboring and non-neighboring 363 root tips, indicating that it was more likely to be located next to root tips assigned to the same 364 OTU in the younger of the root system than in the old (i.e. more clustering, Fig. 4). Clustering of 365 root tips belonging to the same OTU is visualized schematically in Fig. 5. 


\section{Discussion}

\section{Spatial structure within the root system}

369 The ordination analyses showed a clear spatial structure within the root system for the community recovered from fine roots from different parts of the rhizome, with a shift in the fungal community from the youngest end of the rhizome to the older. This pattern was evident

372 although only $32.1 \%$ of the total number of root tips was included in this analysis. Neither

373 weighting functions nor ordination method (DCA or GNMDS) affected the overall structure,

374 therefore it is reasonable to believe that the structure found was not due to mathematical artifacts

375 caused by weighting function or ordination method (Son and Halvorsen 2014). Previous studies have not found any clear structural patterns at finer scales $(<3 \mathrm{~m})$ for the fungal communities of B. vivipara (Mühlmann et al. 2008; Blaalid et al. 2012, 2014; Yao et al. 2013; Botnen et al. 2014), except that Blaalid (2012) observed a spatial auto-correlation below $50 \mathrm{~cm}$ scale. In other studies, spatial heterogeneity and patchiness of fungal communities at finer scales have been observed (Taylor and Bruns 1999; Tedersoo et al. 2003; Lilleskov et al. 2004; Pickles et al. 2012; Blaalid et al. 2014). Several studies have found that some fungal species exhibit preference for certain microhabitats in the soil (Taylor and Bruns 1999; Tedersoo et al. 2003, 2008; Genney 383 et al. 2006; Buée et al. 2007). However, for EcM species the scale that species are occupying as 384 root tips may be very different from the scale species are occupying as mycelium (Genney et al. 385 2006; Pickles et al. 2012). Yoshida et al. (2014) revealed a tendency towards a spatial clustering 386 of individual EcM species within the root systems of T. diversifolia seedlings, but the present 387 study is to our knowledge the first time a clear spatial structuring at such a fine scale of the rootassociated fungal community is shown for a full grown plant. richness and diversity of the fungal community also changed throughout the root system. For the

In addition to a shift in the overall root-associated fungal community throughout the root system, EcM fungi, exploration type recovered from fine roots also changed from the younger to the older end of the rhizome. The higher abundance of the contact exploration type found in association with the older end of the rhizome was mainly because OTU4 (Russula pascua), the only OTU assigned to this exploration type was more abundant in this part of the root system. 
397 From the $\chi^{2}$-tests, the structuring of the fungal community was evident at a scale ranging from 398 rhizome parts to individual OTUs. OTU1 (Pseudotomentella sp.), although common throughout 399 the whole root system, was more common in the youngest part of the root system than expected 400 by a random distribution. Although at very different spatial scales, Pseudotomentella has been 401 reported to be observed in younger rather than older forest stands (Kyaschenko et al. 2017), 402 suggesting that it may be a weak competitor. OTU3 (Cortinarius sp.) and OTU4 (Russula 403 subrubens) were relatively more abundant in $\mathrm{R} 3$ and $\mathrm{R} 4$. There might be a succession with age 404 of the root system of root-associated fungi within this root system, similar to what is observed at 405 larger scales.

Priority effects?

408 Priority effects have been observed for several species (Alford and Wilbur 1985; Shorrocks and 409 Bingley 1994), including fungi (Kennedy and Bruns 2005; Yoshida et al. 2014; Hiscox et al. 410 2015). For EcM fungi, the priority effect may be observed as clustering of species within a root 411 system, and a dominance of one or a few species, when the timing of arrival of species is 412 unknown (Yoshida et al. 2014). Although only relative distances are recorded in this study, root 413 tips located in sequence on a branch from a fine root were assumed to be closer in proximity to 414 each other than root tips not located on the same branch or on other fine roots. With these 415 assumptions, it was possible to investigate whether or not the neighbourhood of a root tip 416 included several root tips sharing the same OTU (i.e. clusters). There was a higher mean of 417 neighbouring root tips sharing the same OTU than non-neighbouring root tips, indicating 418 clustering of species, and this was particularly evident in the younger part of the rhizome where 419 more than half of all neighbouring root tips shared OTUs (Fig. 3). The mean number of 420 neighbouring root tips sharing OTUs was much higher in the younger parts (R1 and R2) than in 421 the older parts (R3 and R4). In the oldest part of the root system almost none of the investigated 422 neighbouring root tips shared an OTU (estimated mean = 0.9, Fig. 4).

424 According to priority effects, colonisation in the early stages of root formation may result in clusters 425 of species. Clusters similar to what Yoshida et al. (2014) found in their study were observed only in 426 the youngest parts of this particular root system, but in the older parts no evident clustering of OTUs 427 was observed. As freshly formed fine roots were only observed in the youngest part of the rhizome, 
428 we deem it likely that these clusters are a result of priority effects rather than competition. This was

429 seen from the T-test on strict neighbours, but also for the neighbourhood (i.e. branches on fine roots, 430 data not shown). Similarly, Pickles et al. (2012) found that in a mature forest system, competition, or 431 processes generating similar patterns, was determining the fine scale distribution of EcM fungi. It is 432 possible that succession and competition play a more prominent role in structuring the fungal 433 community in more established root systems compared to younger root systems, where more newly 434 formed roots are available colonisation.

Richness and community composition

437 A total of 41 root-associated fungal OTUs were recovered from the analysed root system.

438 Although only $32.1 \%$ of all root tips were successfully sequenced in the present study, this

439 accords well with previous studies on root-associated fungi in B. vivipara using high throughput

440 sequencing of entire root systems (e.g. Blaalid et al. 2012, 2014; Yao et al. 2013; Botnen et al.

441 2014), where ranges of root-associated OTUs generally fall between (10-159). However, as

442 indicated by the accumulation curve, the root system most likely possesses a somewhat richer

443 community than we were able to recover. Few other studies have been conducted where the entire

444 mycobiome of whole root systems has been profiled root tip by root tip. Bahram et al. (2011)

445 recovered $122 \mathrm{EcM}$ fungal species in 103 root samples from an extensively sampled root system of a

446 P. tremula individual, while Yoshida et al. (2014) recovered 40 EcM species when mapping the

447 entire EcM community of three T. diversifolia seedlings.

449 The OTUs followed a log-normal distribution, where a few OTUs were common and the 450 majority was represented by ten or less root tips (Fig. 1a). Similar to these results, Yoshida et al. 451 (2014) observed that the majority of fungal species recovered from three seedlings of T. diversifolia 452 occupied less than ten root tips, with only one or a few species dominating the root system of a 453 seedling. Bahram et al. (2011) observed the same pattern, where a single species was found in 59.2 $454 \%$ of all samples. Dominance of one or a few species seems to be common in root-associated fungal 455 communities.

456 In accordance with earlier studies on the fungal community of B. vivipara (Massicotte et al. 1998; 457 Mühlmann et al. 2008; Brevik et al. 2010; Blaalid et al. 2012, 2014; Yao et al. 2013; Botnen et al. 458 2014), most of the detected OTUs were EcM fungi. The two most dominant orders, Thelephorales 459 and Agaricales, are well established as EcM forming linages (Smith \& Read 2008; Tedersoo et al. 
2010). In contrast to the abovementioned studies of B. vivipara, only typical ECM root tips or tips with signs of fungal colonization were investigated, and a dominance of ECM fungi is therefore not surprising. The order Thelephorales accounted for more than half of all the successfully sequenced root tips. Thelephorales is found to be among the most common orders in several studies in artic and alpine EcM communities (e.g. Clemmensen \& Michelsen 2006; Mühlmann et al. 2008; Bjorbækmo et al. 2010; Timling et al. 2012; Blaalid et al. 2012; Yao et al. 2013; Botnen et al. 2014; Morgado et al. 2015; Mundra \& Halvorsen 2015), suggesting that several species within this order tolerate harsh climatic and environmental conditions. All of the most common OTUs, Pseudotomentella sp. (OTU1), Thelephorales sp.1 (OTU2), Cortinarius sp. (OTU3, OTU15, OTU23) and Russula (OTU4) were ectomycorrhizal.

Due to problems with contaminants, a strict criterion for including OTUs in downstream analysis was used: OTUs with hits to all yeasts and/or fungi that had not previously been reported from plant roots were excluded with two exceptions: OTU12 (99.9\% match to Sarcoleotia globosa) and OTU19 (99.0\% match to Clitocybe subditopoda). Clitocybe is generally considered a saprotrophic genus, OTU19 clustered together with ITS sequences with affinity to Tricholoma, a known EcM genus, in the initial NJ tree and Clitocybe subidtopoda has been found to be unrelated to the core Clitocybe group (Alvarado et al. 2015). Hence, it might be a root-associated fungus. S. globosa within Geoglossales forms small, stipitate fruit bodies, and due to its small size, most likely overlooked in field surveys. Its distribution has previously been described as arctic and sub-arctic, although stretching as far south as cool temperate (Jumpponen et al. 1997). It is often found in open vegetation and on disturbed ground (Schumacher and Sivertsen 1987; Jumpponen et al. 1997), which fits the sample site of the B. vivipara root system used in this study. It has been suggested to have saprotrophic lifestyle, however, in a survey of stable isotopes, a relatively high enrichment in $\delta^{15} \mathrm{~N}$ was shown for species within Geoglossales (Griffith et al. 2002). Additionally, several collections have been made where S. globosa has been found in association with mosses or the ericaceous Empetrum nigrum (Schumacher and Sivertsen 1987). Extreme isotopic profiles for $\delta^{15} \mathrm{~N}$ and $\delta^{13} \mathrm{C}$ and associations with plants may suggest a possibility of unknown biotrophic lifestyles (also discussed in Tedersoo et al. 2010). 


\section{Conclusion}

491 For the first time, an attempt to map the entire root-associated fungal community of an adult EcM

492 plant was conducted. Although the direct PCR approach was not suitable for recovering the entire

493 fungal community associated with this plant, and only a third of the root-associated fungal

494 community could be identified, a diverse and spatially structured fungal community was discovered.

495 Indications of possible priority effects were restricted to the younger parts of the root system,

496 whereas in the older parts, we did not observe these patterns, suggesting that competition may be

497 more important in structuring mature root systems. This study provides a first glimpse into the fine

498 scale structuring of the root-associated fungal community of an adult EcM plant. 


\section{Conflict of interest}

501 The authors declare that they have no conflict of interest.

\section{Authors' contribution:}

503 All authors have contributed to the completion of the article. The main ideas and design of the

504 study was done by TC, HK and ET. Sampling and laboratory work was conducted by mainly ET, 505 but also ABA and UV. ABA contributed substantially in ideas on analyses of the data. Drafting 506 and writing of the manuscript was mainly performed by ET, but all authors (ABA, UV, AKB, IS, 507 TC, HK) contributed substantially to supervision, ideas and discussion of the results in the 508 writing process.

\section{Acknowledgments}

510 We would like to acknowledge Cecilie Mathiesen for an excellent effort in preparation of 511 samples for sequencing, Rune Halvorsen for valuable input on ordination methods and Erlend Y.

512 Fines for assistance in graphical design and excellent drawing. We would like to thank the 513 University of Oslo for funding this project. 


\section{References}

516 Agerer R (2001) Exploration types of ectomycorrhizae: A proposal to classify ectomycorrhizal mycelial systems according to their patterns of differentiation and putative ecological importance. Mycorrhiza 11:107-114. doi: 10.1007/s005720100108

Agerer R, Rambold G DEEMY - An Information System for Characterization and Determination of Ectomycorrhizae. www.deemy.de - München, Ger.

Alford R, Wilbur H (1985) Priority effects in experimental pond communities: competition between Bufo and Rana. Ecology 66:1097-1105

Alva V, Nam S-Z, Söding J, Lupas AN (2016) The MPI bioinformatics toolkit as an integrative platform for advanced protein sequence and structure analysis. Nucleic Acids Res 44:W410-W415. doi: 10.1093/nar/gkw348

Alvarado P, Moreno G, Vizzini A, et al (2015) Atractosporocybe, Leucocybe and Rhizocybe : three new clitocyboid genera in the Tricholomatoid clade (Agaricales) with notes on Clitocybe and Lepista. Mycologia 107:123-136. doi: 10.3852/13-369

Anderson IC, Genney DR, Alexander IJ (2014) Fine-scale diversity and distribution of ectomycorrhizal fungal mycelium in a Scots pine forest. New Phytol 201:1423-1430. doi: 10.1111/nph.12637

Averill C, Turner BL, Finzi AC (2014) Mycorrhiza-mediated competition between plants and decomposers drives soil carbon storage. Nature 505:543-545. doi: 10.1038/nature12901

Bahram M, Peay KG, Tedersoo L (2015) Local-scale biogeography and spatiotemporal variability in communities of mycorrhizal fungi. New Phytol 205:1454-1463. doi: 10.1111/nph.13206

Bahram M, Põlme S, Kõljalg U, Tedersoo L (2011) A single European aspen (Populus tremula) tree individual may potentially harbour dozens of Cenococcum geophilum ITS genotypes and hundreds of species of ectomycorrhizal fungi. FEMS Microbiol Ecol 75:313-320. doi: 10.1111/j.1574-6941.2010.01000.x

Bergemann SE, Miller SL (2002) Size, distribution, and persistence of genets in local populations of the late-stage ectomycorrhizal basidiomycete, Russula brevipes. New Phytol 156:313-320. doi: 10.1046/j.1469-8137.2002.00507.x

Bjorbækmo M, Carlsen T, Brysting A, et al (2010) High diversity of root associated fungi in both alpine and arctic Dryas octopetala. BMC Plant Biol 10:244. doi: 10.1186/1471-2229$10-244$

Blaalid R, Carlsen T, Kumar S, et al (2012) Changes in the root-associated fungal communities along a primary succession gradient analysed by 454 pyrosequencing. Mol Ecol 21:18971908. doi: 10.1111/j.1365-294X.2011.05214.X

Blaalid R, Davey ML, Kauserud H, et al (2014) Arctic root-associated fungal community composition reflects environmental filtering. Mol Ecol 23:649-659. doi: 
553

554

555

556

557

558

559

560

561

562

563

564

565

566

567

568

569

570

571

572

573

574

575

576

577

578

579

580

581

582

583

584

585

586

587

Bogar LM, Peay KG (2017) Processes maintaining the coexistence of ectomycorrhizal fungi at a fine spatial scale. In: Tedersoo L (ed) Biogeography of Mycorrhizal Symbiosis. Springer International Publishing, Cham, pp 79-105

Botnen S, Vik U, Carlsen T, et al (2014) Low host specificity of root-associated fungi at an Arctic site. Mol Ecol 23:975-985. doi: 10.1111/mec.12646

Brevik A, Moreno-Garcia J, Wenelczyk J (2010) Diversity of fungi associated with Bistorta vivipara (L.) Delarbre root systems along a local chronosequence on Svalbard. Agarica 29:15-26. doi: ISSN 0800-1820

Brundrett MC, Tedersoo L (2018) Evolutionary history of mycorrhizal symbioses and global host plant diversity. New Phytol 220:1108-1115. doi: 10.1111/nph.14976

Buée M, Courty PE, Mignot D, Garbaye J (2007) Soil niche effect on species diversity and catabolic activities in an ectomycorrhizal fungal community. Soil Biol Biochem 39:19471955. doi: 10.1016/j.soilbio.2007.02.016

Carlsen TA (2002) Molecular diversity of root endophytes in an alpine Bistorta vivipara Kobresia myosuroides tundra plant community (Cand. scient. thesis). University of Oslo

Clemmensen KE, Bahr A, Ovaskainen O, et al (2013) Roots and associated fungi drive longterm carbon sequestration in boreal forest. Science 339:1615-1618. doi:

$10.1126 /$ science. 1231923

Clemmensen KE, Finlay RD, Dahlberg A, et al (2015) Carbon sequestration is related to mycorrhizal fungal community shifts during long-term succession in boreal forests. New Phytol 205:1525-1536. doi: 10.1111/nph.13208

Clemmensen KE, Michelsen A (2006) Integrated long-term responses of an arctic-alpine willow and associated ectomycorrhizal fungi to an altered environment. Can J Bot 84:831-843. doi: $10.1139 / \mathrm{b} 06-039$

Clymo RS (1980) Preliminary survey of the peat-bog Hummel Knowe moss using various numerical methods. Vegetatio 42:129-148

Dahlberg A, Stenlid J (1994) Size, distribution and biomass of genets in populations of Suillus bovinus (L.: Fr.) Roussel revealed by somatic incompatibility. New Phytol 128:225-234. doi: 10.1111/j.1469-8137.1994.tb04006.x

Diggle PK (1997) Extreme preformation in alpine Polygonum viviparum: an architectural and Developmental Analysis. Am J Bot 84:154-169. doi: 10.2307/2446077

Eriksen M, Bjureke KE, Dhillion SS (2002) Mycorrhizal plants of traditionally managed boreal grasslands in Norway. Mycorrhiza 12:117-123. doi: 10.1007/s00572-002-0165-x

Gardes M, Bruns TD (1993) ITS primers with enhanced specificity for basidiomycetes-application to the identification of mycorrhizae and rusts. Mol Ecol 2:113-8. doi: 
Genney DR, Anderson IC, Alexander IJ (2006) Fine-scale distribution of pine ectomycorrhizas and their extramatrical mycelium. New Phytol 170:381-390. doi: 10.1111/j.14698137.2006.01669.x

Jonsell B (ed) (2000) Flora Nordica 1. Ber, Stockholm

Jumpponen A, Weber NS, Trappe JM, Cázares E (1997) Distribution and ecology of the ascomycete Sarcoleotia globosa in the United States. Can J Bot 75:2228-2231. doi: 10.1139/b97-933\#.W78K7PmYOM8

Kauserud H, Kumar S, Brysting AK, et al (2012) High consistency between replicate 454 pyrosequencing analyses of ectomycorrhizal plant root samples. Mycorrhiza 22:309-315. doi: 10.1007/s00572-011-0403-1

Kendall MG (1938) A new measure of rank correlation. Biometrika 30:81-93. doi: 10.1093/biomet/30.1-2.81

Kennedy PG, Bruns TD (2005) Priority effects determine the outcome of ectomycorrhizal competition between two Rhizopogon species colonizing Pinus muricata seedlings. New Phytol 166:631-638. doi: 10.1111/j.1469-8137.2005.01355.x

Kennedy PG, Higgins LM, Rogers RH, Weber MG (2011) Colonization-competition tradeoffs as a mechanism driving successional dynamics in ectomycorrhizal fungal vommunities. PLoS One 6:e25126. doi: 10.1371/journal.pone.0025126

Kennedy PG, Peay KG, Bruns TD (2009) Root tip competition among ectomycorrhizal fungi: Are priority effects a rule or an exception? Ecology 90:2098-2107. doi: 10.1890/08-1291.1

Kõljalg U, Nilsson RH, Abarenkov K, et al (2013) Towards a unified paradigm for sequence- 
Kruskal JB (1964) Nonmetric multidimensional scaling: A numerical method. Psychometrika 29:115-129. doi: 10.1007/BF02289694

Kruskal WH (1958) Ordinal measures of association. J Am Stat Assoc 53:814. doi:

Kyaschenko J, Clemmensen KE, Hagenbo A, et al (2017) Shift in fungal communities and

Lindahl BD, Ihrmark K, Boberg J, et al (2007) Spatial separation of litter decomposition and mycorrhizal nitrogen uptake in a boreal forest. New Phytol 173:611-620. doi: 10.1111/j.1469-8137.2006.01936.x

Massicotte HB, Melville LH, Peterson RL, Luoma DL (1998) Anatomical aspects of field ectomycorrhizas on Polygonum viviparum (Polygonaceae) and Kobresia bellardii (Cyperaceae). Mycorrhiza 7:287-292. doi: 10.1007/s005720050194

Miyamoto Y, Sakai A, Hattori M, Nara K (2015) Strong effect of climate on ectomycorrhizal fungal composition: evidence from range overlap between two mountains. ISME J 9:18701879. doi: 10.1038/ismej.2015.8

Morgado LN, Semenova TA, Welker JM, et al (2015) Summer temperature increase has distinct effects on the ectomycorrhizal fungal communities of moist tussock and dry tundra in Arctic Alaska. Glob Chang Biol 21:959-972. doi: 10.1111/gcb.12716

Mühlmann O, Bacher M, Peintner U (2008) Polygonum viviparum mycobionts on an alpine primary successional glacier forefront. Mycorrhiza 18:87-95. doi: 10.1007/s00572-0070156-z

Mundra S, Bahram M, Tedersoo L, et al (2015a) Temporal variation of Bistorta viviparaassociated ectomycorrhizal fungal communities in the High Arctic. Mol Ecol 24:62896302. doi: $10.1111 / \mathrm{mec} .13458$

Mundra S, Halvorsen R, Kauserud H, et al (2015b) Arctic fungal communities associated with roots of Bistorta vivipara do not respond to the same fine-scale edaphic gradients as the aboveground vegetation. New Phytol 205:1587-1597. doi: 10.1111/nph.13216

Oksanen J, Blanchet F, Kindt R, et al (2013) vegan: Community Ecology Package 
660

661

662

663

664

665

666

667

668

669

670

671

672

673

674

675

676

677

678

679

680

681

682

683

684

685

686

687

688

689

690

691

692

693

694

695

696

Peay KG, Kennedy PG, Bruns TD (2011) Rethinking ectomycorrhizal succession: Are root density and hyphal exploration types drivers of spatial and temporal zonation? Fungal Ecol 4:233-240. doi: 10.1016/j.funeco.2010.09.010

Pickles BJ, Genney DR, Anderson IC, Alexander IJ (2012) Spatial analysis of ectomycorrhizal fungi reveals that root tip communities are structured by competitive interactions. Mol Ecol 21:5110-5123. doi: 10.1111/j.1365-294X.2012.05739.X

R Core Team (2017) R: A language and environment for statistical computing. R Foundation for Statistical Computing, Vienna, Austria.

Schumacher T, Sivertsen S (1987) Sarcoleotia globosa (Sommerf.: Fr.) Korf, Taxonomy, ecology and distribution. In: Laursen GA, Ammirati JF, Redhead SA (eds) Arctic and Alpine Mycology II. Springer US, Boston, MA, pp 163-176

Shorrocks B, Bingley M (1994) Priority effects and species coexistence: Experiments with fungal-breeding Drosophila. J Anim Ecol 63:799. doi: 10.2307/5257

Smith SE, Read D (2008) Mycorrhizal Symbiosis, 3rd edn. Elsevier, London

Son TC van, Halvorsen R (2014) Multiple parallel ordinations: the importance of choice of ordination method and weighting of species abundance data. Sommerfeltia 37:1-37. doi: 10.2478/som-2014-0001

Sterkenburg E, Clemmensen KE, Ekblad A, et al (2018) Contrasting effects of ectomycorrhizal fungi on early and late stage decomposition in a boreal forest. ISME J 12:2187-2197. doi: $10.1038 / \mathrm{s} 41396-018-0181-2$

Talbot JM, Bruns TD, Taylor JW, et al (2014) Endemism and functional convergence across the North American soil mycobiome. Proc Natl Acad Sci 111:6341-6346. doi: 10.1073/pnas.1402584111

Taylor DL, Bruns TD (1999) Community structure of ectomycorrhizal fungi in a Pinus muricata forest: minimal overlap between the mature forest and resistant propagule communities. Mol Ecol 8:1837-1850. doi: 10.1046/j.1365-294x.1999.00773.x

Tedersoo L, Hallenberg N, Larsson K (2003) Fine scale distribution of ectomycorrhizal fungi and roots across substrate layers including coarse woody debris in a mixed forest. New Phytol 159:153-165. doi: 10.1046/j.0028-646x.2003.00792.x

Tedersoo L, May TW, Smith ME (2010) Ectomycorrhizal lifestyle in fungi: global diversity, distribution, and evolution of phylogenetic lineages. Mycorrhiza 20:217-63. doi: 10.1007/s00572-009-0274-X

Tedersoo L, Suvi T, Jairus T, Kõljalg U (2008) Forest microsite effects on community composition of ectomycorrhizal fungi on seedlings of Picea abies and Betula pendula. Environ Microbiol 10:1189-1201. doi: 10.1111/j.1462-2920.2007.01535.x

Tenenbaum JB (2000) A global geometric framework for nonlinear dimensionality reduction. Science 290:2319-2323. doi: 10.1126/science.290.5500.2319 
Timling I, Dahlberg A, Walker D, et al (2012) Distribution and drivers of ectomycorrhizal fungal communities across the North American Arctic. Ecosphere 3(11):1-25

van der Linde S, Suz LM, Orme CDL, et al (2018) Environment and host as large-scale controls of ectomycorrhizal fungi. Nature 558:243-248. doi: 10.1038/s41586-018-0189-9

van der Maabel E (1979) Transformation of cover-abundance values in phytosociology and its effects on community similarity. Vegetatio 39:97-114. doi: https://doi.org/10.1007/BF00052021

White T, Bruns T, J T (1990) Analysis of phylogenetic relationships by amplification and direct sequencing of ribosomal RNA genes. In: Innis M, Gelfand D, Sninsky J, White T (eds) PCR Protocols: A Guide to Methods and Applications. Academic Press, New York, pp 315-322

Yao F, Vik U, Brysting AK, et al (2013) Substantial compositional turnover of fungal communities in an alpine ridge-to-snowbed gradient. Mol Ecol 22:5040-52. doi: $10.1111 / \mathrm{mec} .12437$

Yoshida N, Son J a, Matsushita N, et al (2014) Fine-scale distribution of ectomycorrhizal fungi colonizing Tsuga diversifolia seedlings growing on rocks in a subalpine Abies veitchii forest. Mycorrhiza 24:247-57. doi: 10.1007/s00572-013-0535-6 


\section{Figure Captions}

718 Fig.1 Schematic drawing of B. vivipara, with its unbranched rhizome with fine roots and ECM root tips. R1-R4 are

719 the approximate parts the rhizome was dissected into, and subsequently used as age groups, where R1 is the

720 youngest part of the root system, and R4 is the oldest. Figure credit: Erlend Y. Fines

721 Fig 2 Barplot of the frequency of the 26 non-singleton OTUs (A), where a few OTUs are very frequent, while most

722 OTUs are rare, and the species accumulation curve (B) using fine roots as samples curve does not level off.

723 Fig. 3 GNMDS ordination diagram of power-weighted data $(w=0.5)$ for all analyzed root tips, where fine roots are

724 used as sites. The diagram is showing a gradient from the younger (R1) part of the root system through to the older

725 (R4). Arrows depict significant variables fitted onto the ordination diagrams using envar function in the vegan

726 package (Oksanen et al. 2013). position=position of fine roots along the rhizome, diversity=Shannon's H calculated

727 per fine roots, richness=richness per fine root, length=relative length of fine roots, branching=number of branches

728 per fine root, no_root_tip=number of root tips per fine root. Assigned exploration types are: contact, short smooth

729 (sh_smooth), medium smooth (med_smooth) and additionally, hydrophilic for hydrophilic exploration types

730 Fig. 4 The proportion of pairs of root tips sharing the same OTU per age class (R1, R2, R3 and R4). Shared OTU

731 between pairs of root tips is shown in dark grey, and pairs of root tips not sharing OTU is shown in light grey. The

732 proportion of shared OTUs between pairs of root tips is larger in R1 and declines throughout the age classes

733 Fig. 5 Schematic map of the entire root system the B. vivipara individual. R1-4 are the four parts the rhizome was

734 dissected into and subsequently used as age classes in the analyses of the root system. Fine roots and branches are

735 scaled in lengths relative to each other based on photographic measurements, but the spatial distance between fine

736 roots and root tips are not realistically depicted in the map. Root tip colour indicate OTUs as described in the legend,

737 light grey root tips were not identified

\section{Supplementary Information}

739 File: Table S1_S2_S3.pdf

740 Table S1 List of all sequence reads (No. of seqs.) that were omitted from downstream analyses, because of either

741 appearing in negative controls, or not described as a root-associated fungus

742 Table S2 List of singleton OTUs, their hit against the UNITE database (including INSD and Environmetal

743 sequences), with their reference sequence ID (UNITE Top Hit) and assigned species hypothesis (SH)

744 Table S3 Variables fitted onto the GNMDS ordination diagram, Fig. 3, using the envar function in the vegan 745 package in $\mathrm{R}$ with 99 permutations. 


\section{$747 \quad$ File: FigS1.tif}

748 Fig. S1 Multiple parallel ordinations (MPO) using DCA (A, B, C) and GNMDS (D, E, F) with raw (A, D), power-

749 weighted $(\mathrm{w}=0.5, \mathrm{~B}, \mathrm{E})$ and presence/absence $(\mathrm{C}, \mathrm{F})$ data using fine roots as sites. All ordination methods and

750 weighting functions show similar point configuration, supporting a strong gradient in the dataset. Arrows depicts

751 significant variables fitted onto the ordination diagrams using envar function in the vegan package (Oksanen et al.

752 2013). position=postion of fine root along the rhizome, diversity=Shannon's H calculated per fine root,

753 richnes=richness per fine root, length=relative length of fine root, branch=number of branches per fine root,

754 no_root_tip=number of root tips per fine root, no_exp_types=number of exploration type per fine root. Assigned

755 exploration types are: contact, short smooth (sh_smooth), medium smooth (med_smooth) and additionally,

756 hydrophilic for hydrophilic exploration types

\section{$757 \quad$ File: FigS2.tif}

\section{File: FigS2.tif}

759 Fig. S2 Photograph of one of the fine roots from the B. vivipara root system, showing how root tips are growing

760 from fine roots and branches from the main fine roots. Because the real orientation of the main fine roots and

761 branches in the soil is unknown, we assigned the root tips to three distance classes; neighbour, adjacent and non-

762 neighbour. We assumed that, for instance, A and B were located in very close proximity in the soil (i.e. strict

763 neighbours), and that $\mathrm{C}$, on the same branch, is closer to $\mathrm{A}$ and $\mathrm{B}$ (i.e. adjacent) than it was to $\mathrm{D}$, which was most

764 likely located further away from A, B and C (i.e. non-neighbour) 
Table 1. The distribution of fine roots and root tips throughout the root system. R1-R4 represent the different rhizome parts or age classes in the root system (see Fig. 1). The \% coverage is the percent of how many root tips of the total amount sampled root tips that passed quality control and produced an acceptable sequence. Richness is the number of OTUs per age class; Shannon's H is used as a diversity measure.

\begin{tabular}{llllll}
\hline & R1 & R2 & R3 & R4 & Sum \\
\hline No. of fineroots & 13 & 3 & 10 & 3 & 29 \\
No. of root tips & 1317 & 32 & 637 & 316 & 2302 \\
$\begin{array}{l}\text { No. of successfully } \\
\text { sequenced root tips }\end{array}$ & 448 & 17 & 193 & 83 & 741 \\
\% coverage & 34.02 & 53.13 & 30.98 & 26.27 & 100 \\
Richness & 27 & 7 & 25 & 19 & \\
Shannon's H & 1.978 & 1.809 & 2.368 & 2.552 & \\
& & & & &
\end{tabular}


Table 2. Summary of all non-singleton OTUs, their abundance and their corresponding top hit in to sequence ID in UNITE (Kõljalg et al. 2013,Unite Top Hit ID) and top hit to species hypothesis (UNITE Top Hit SH). The distribution of OTUs throughout the rhizome's four parts R1, R2, R3 and R4 is given, as well as $\chi^{2}$ test results, degrees of freedom (df) and p-value (only calculated for OTUs with more than 10 observations).

\begin{tabular}{|c|c|c|c|c|c|c|c|c|c|c|c|c|c|}
\hline OTU ID & $\begin{array}{l}\text { \# of } \\
\text { seq. }\end{array}$ & UNITE Top Hit ID/SH & Taxonomy & Identity & Score & $\begin{array}{c}\mathrm{e}- \\
\text { value }\end{array}$ & R1 & $\mathbf{R 2}$ & R3 & $\mathbf{R 4}$ & $\chi^{2}$ & $\begin{array}{l}\text { d } \\
\mathbf{f}\end{array}$ & p-value \\
\hline OTU1 & 265 & LC035237|SH587854.07FU & Pseudotomentella sp. & $98 \%$ & 1142 & 0.0 & 202 & 3 & 83 & 29 & 40.825 & 3 & $<0.001$ \\
\hline OTU2 & 131 & KF514695|SH007290.07FU & Thelephorales sp.1 & $98 \%$ & 1193 & 0.0 & 83 & 3 & 42 & 3 & 15.025 & 3 & 0.002 \\
\hline OTU3 & 67 & KM403065|SH353253.07FU & Cortinarius sp. & $99 \%$ & 1101 & 0.0 & 29 & 2 & 29 & 7 & 9.926 & 3 & 0.019 \\
\hline OTU4 & 46 & AY061705|SH312994.07FU & Russula subrubens & $99 \%$ & 1290 & 0.0 & 12 & 0 & 17 & 16 & 25.244 & 3 & $<0.001$ \\
\hline OTU5 & 38 & GU998390|SH338338.07FU & Helotiales sp.1 & $98 \%$ & 1020 & 0.0 & 23 & 2 & 9 & 4 & 4.679 & 3 & 0.197 \\
\hline OTU6 & 17 & FJ378794|SH317877.07FU & Tomentella sp.1 & $99 \%$ & 1236 & 0.0 & 14 & 0 & 1 & 2 & 5.789 & 3 & 0.166 \\
\hline OTU7 & 22 & JX630403|SH323713.07FU & Serendipita sp.1 & $99 \%$ & 1131 & 0.0 & 22 & 0 & 0 & 0 & 16.454 & 3 & $<0.001$ \\
\hline OTU8 & 18 & KF617590|SH422994.07FU & Sordariales sp. & $99 \%$ & 1054 & 0.0 & 17 & 0 & 1 & 0 & 10.264 & 3 & 0.016 \\
\hline OTU9 & 18 & FJ378858|SH392777.07FU & Lachnum sp. & $99 \%$ & 1029 & 0.0 & 2 & 1 & 7 & 8 & 22.126 & 3 & $<0.001$ \\
\hline OTU10 & 15 & KU176247|SH229647.07FU & Tomentella sp.2 & $99 \%$ & 1191 & 0.0 & 4 & 5 & 0 & 6 & $\begin{array}{r}124.29 \\
6\end{array}$ & 3 & $<0.001$ \\
\hline OTU11 & 11 & GU211939|SH320635.07FU & Sebacinales sp. & $98 \%$ & 1043 & 0.0 & 0 & 0 & 6 & 5 & 17.383 & 3 & $<0.001$ \\
\hline OTU12 & 10 & AY789429|SH419762.07FU & Sarcoleotia globosa & $99 \%$ & 1555 & 0.0 & 0 & 0 & 6 & 4 & 14.665 & 3 & 0.002 \\
\hline OTU13 & 9 & FJ553656|SH290706.07FU & $\begin{array}{l}\text { Cadophora } \\
\text { finlandica }\end{array}$ & $99 \%$ & 1548 & 0.0 & 1 & 0 & 4 & 4 & - & - & - \\
\hline OTU14 & 9 & UDB017644|SH448082.07FU & Laccaria laccata & $99 \%$ & 1144 & 0.0 & 1 & 0 & 5 & 3 & - & - & - \\
\hline OTU15 & 8 & UDB011255SH353127.07FU & Cortinarius sp. 2 & $99 \%$ & 996 & 0.0 & 8 & 0 & 0 & 0 & - & - & - \\
\hline OTU16 & 7 & UDB025680SH417154.07FU & Pulvinula sp. & $99 \%$ & 994 & 0.0 & 7 & 0 & 0 & 0 & - & - & - \\
\hline OTU17 & 8 & KU176240|SH401042.07FU & Phialocephala sp. & $99 \%$ & 1510 & 0.0 & 1 & 1 & 4 & 1 & - & - & - \\
\hline
\end{tabular}




\begin{tabular}{|c|c|c|c|c|c|c|c|c|c|c|c|c|c|}
\hline OTU18 & 9 & EU862204|SH445618.07FU & Clavulina sp. & $100 \%$ & 1269 & 0.0 & 6 & 0 & 2 & 1 & - & - & - \\
\hline OTU19 & 3 & EU697244|SH362287.07FU & $\begin{array}{l}\text { Clitocybe } \\
\text { subditopoda }\end{array}$ & $99 \%$ & 1139 & 0.0 & 0 & 0 & 2 & 1 & - & - & - \\
\hline OTU20 & 3 & FJ378854|SH326471.07FU & Pezoloma ericae & $99 \%$ & 915 & 0.0 & 0 & 0 & 2 & 1 & - & - & - \\
\hline OTU21 & 2 & KP742955|SH596590.07FU & Geoglossum sp. & $94 \%$ & 780 & 0.0 & 0 & 0 & 1 & 1 & - & - & - \\
\hline OTU22 & 2 & JX630752|SH250316.07FU & Thelephora sp. & $99 \%$ & 1148 & 0.0 & 2 & 0 & o & 0 & - & - & - \\
\hline OTU23 & 2 & KJ421158|SH581131.07FU & Cortinarius sp. 3 & $99 \%$ & 1115 & 0.0 & 0 & 0 & 2 & 1 & - & - & - \\
\hline OTU24 & 2 & KF218970|SH341492.07FU & Sistotrema sp. & $99 \%$ & 1200 & 0.0 & 0 & 1 & 0 & 1 & - & - & - \\
\hline OTU25 & 2 & HQ850136|SH338142.07FU & Helotiales sp.2 & $99 \%$ & 946 & 0.0 & 2 & 0 & 0 & 0 & - & - & - \\
\hline OTU26 & 2 & KC965976|SH404461.07FU & Helotiales sp 3 & $99 \%$ & 949 & 0.0 & 1 & 0 & 1 & 0 & - & - & - \\
\hline
\end{tabular}




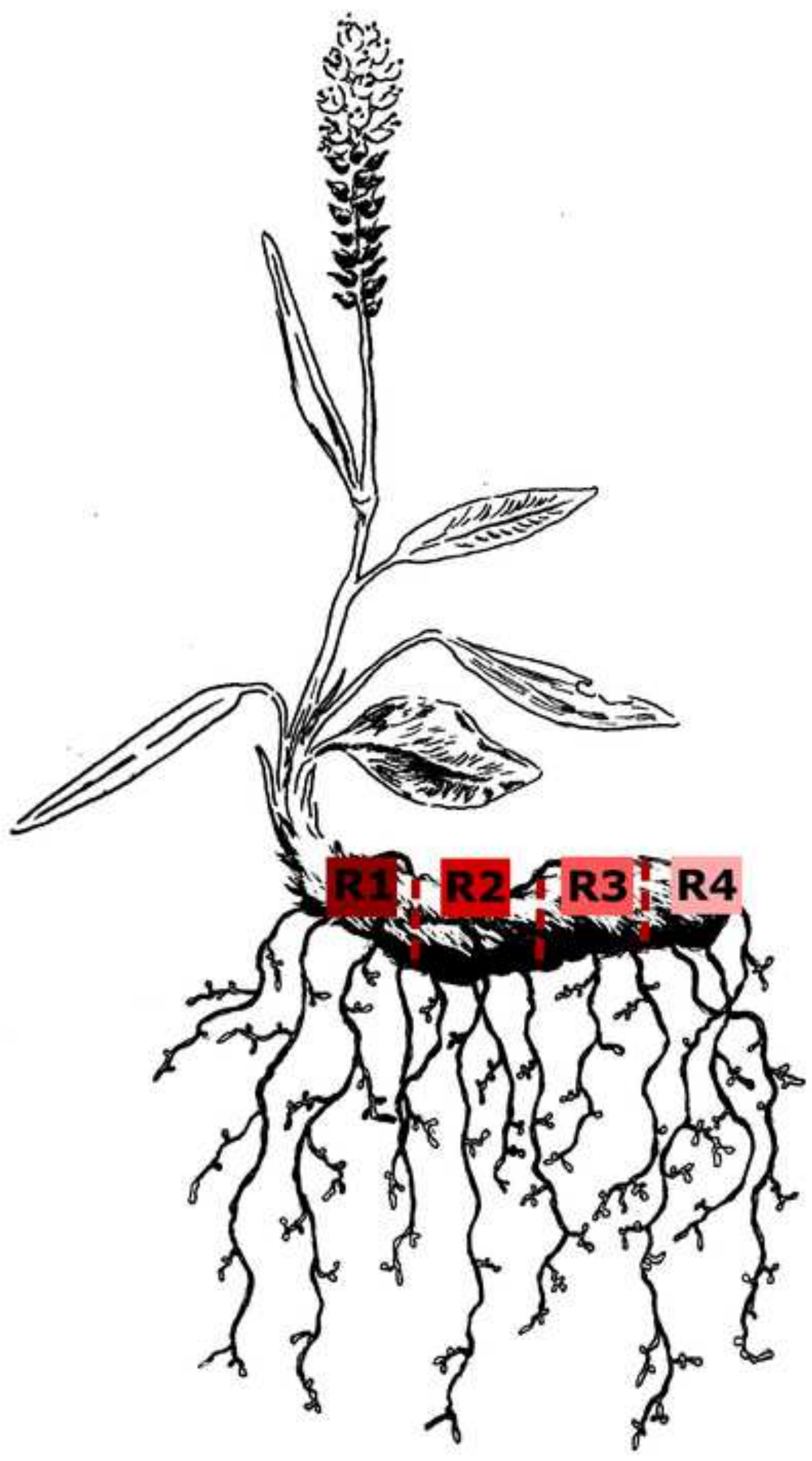


Fig. 2

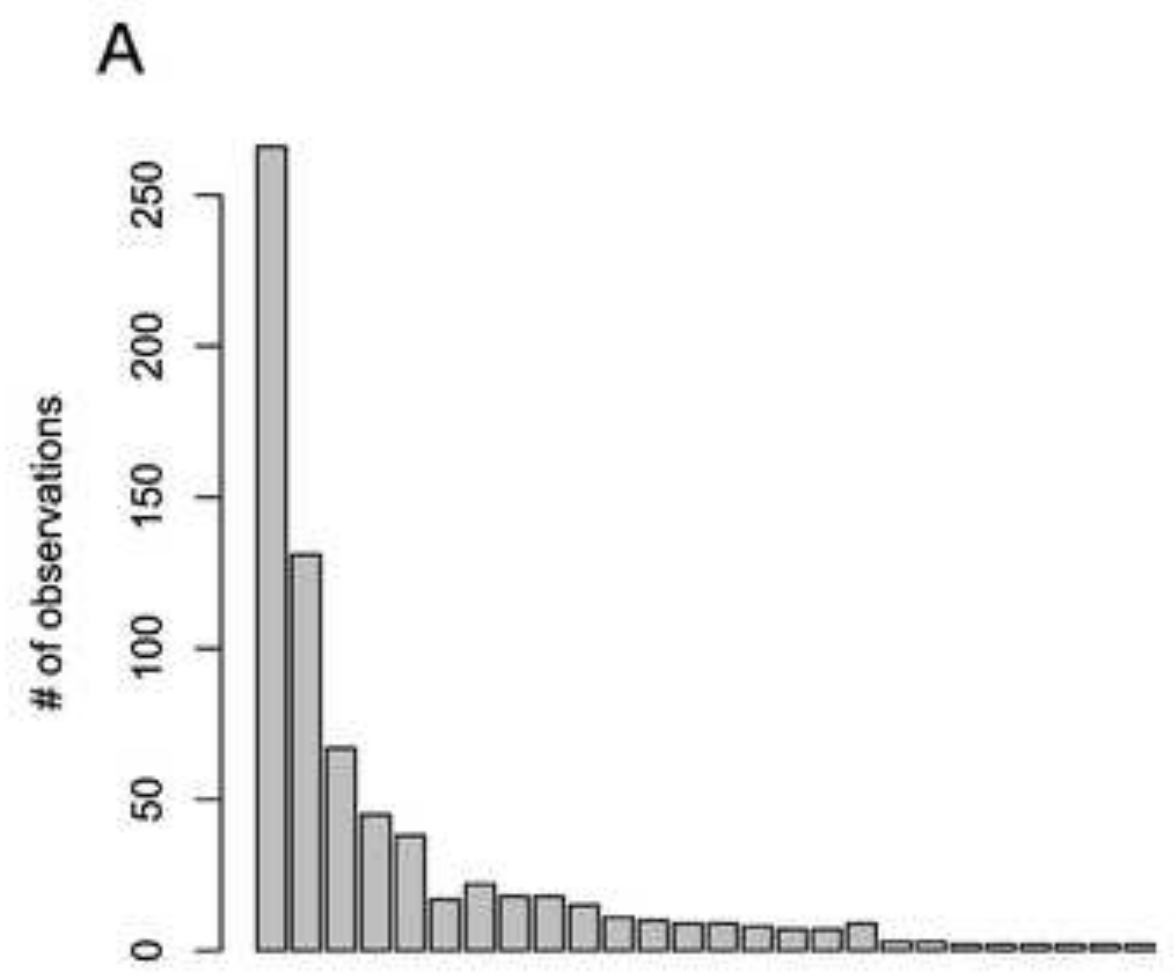

Non-singelton OTUs

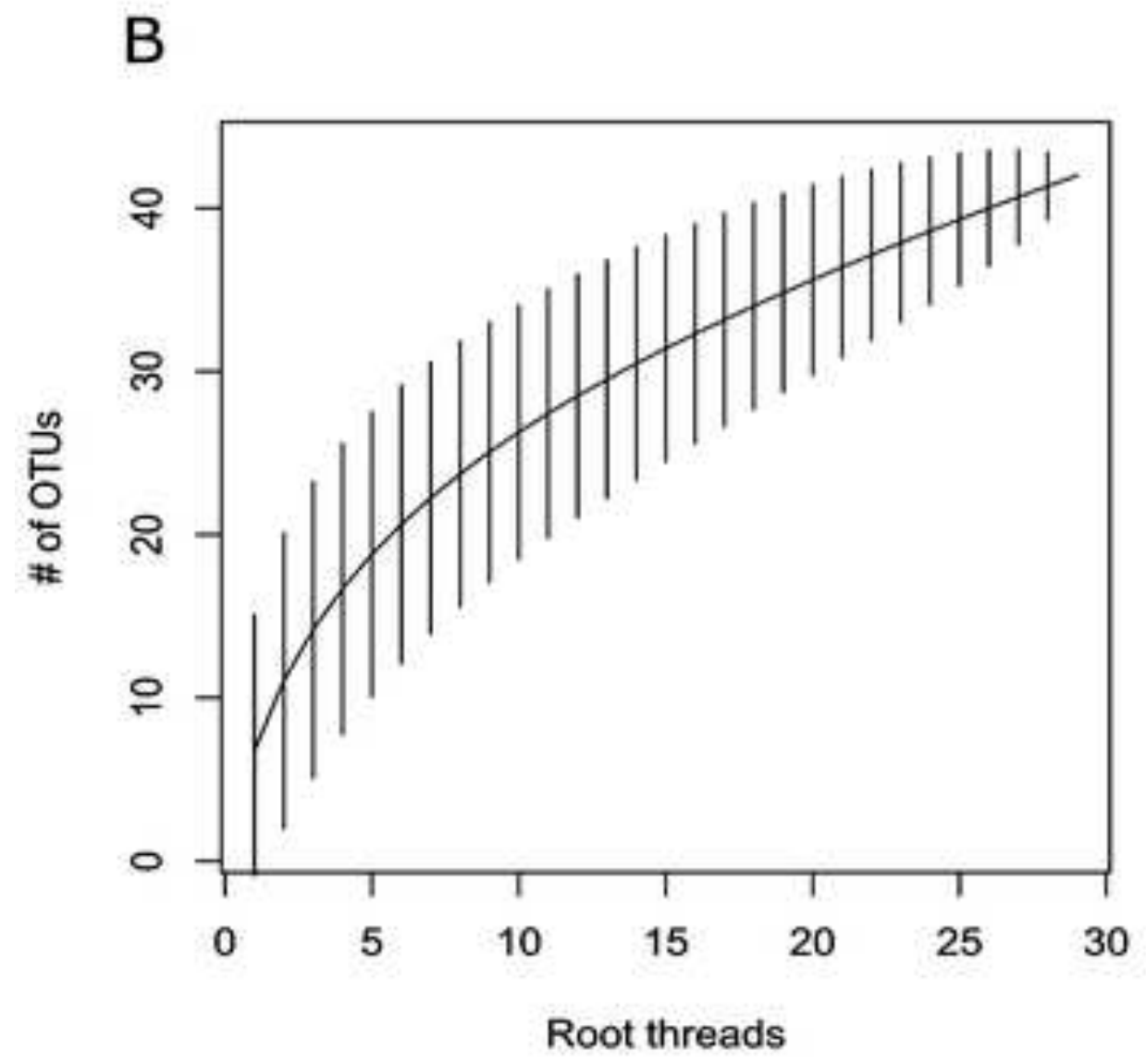




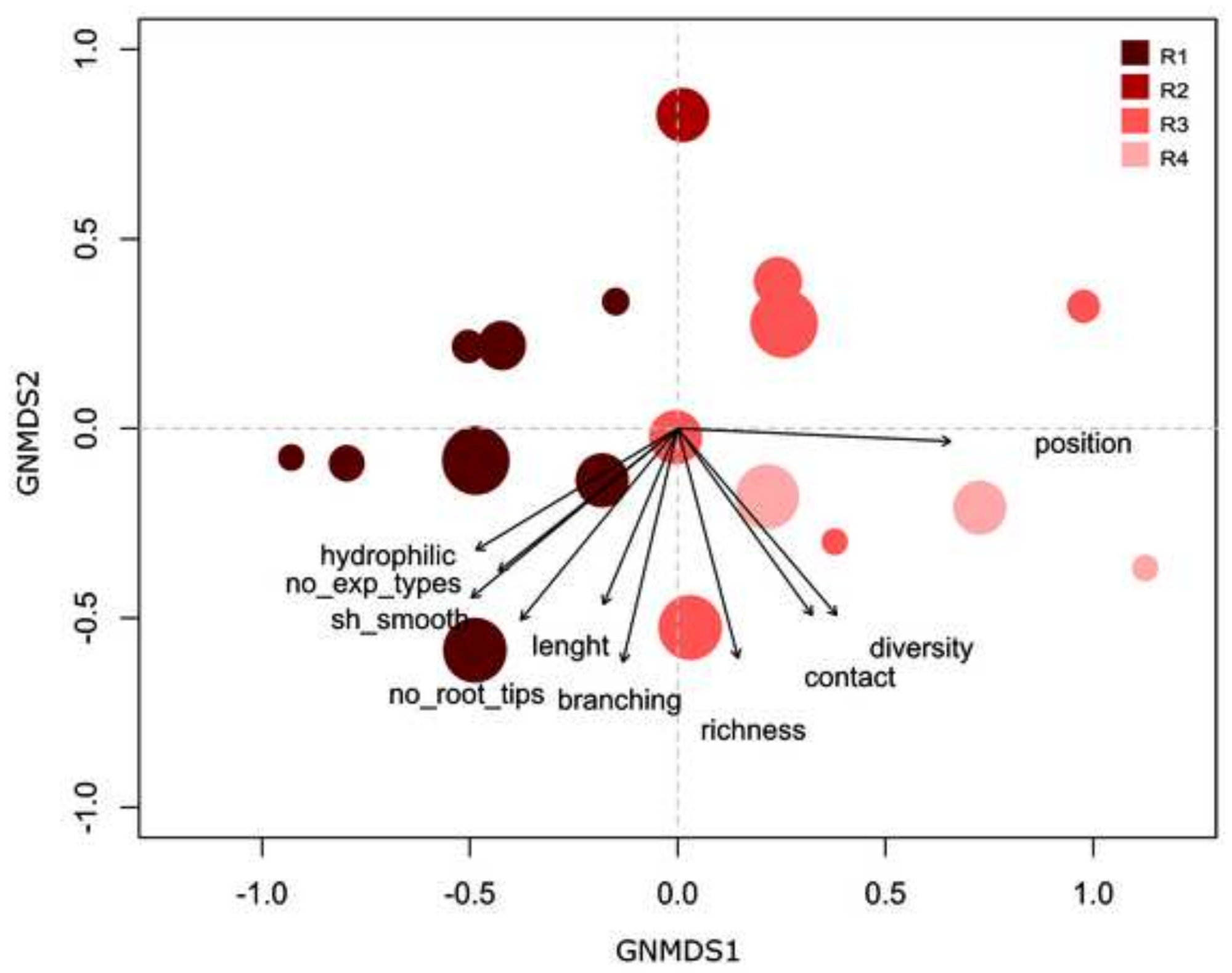




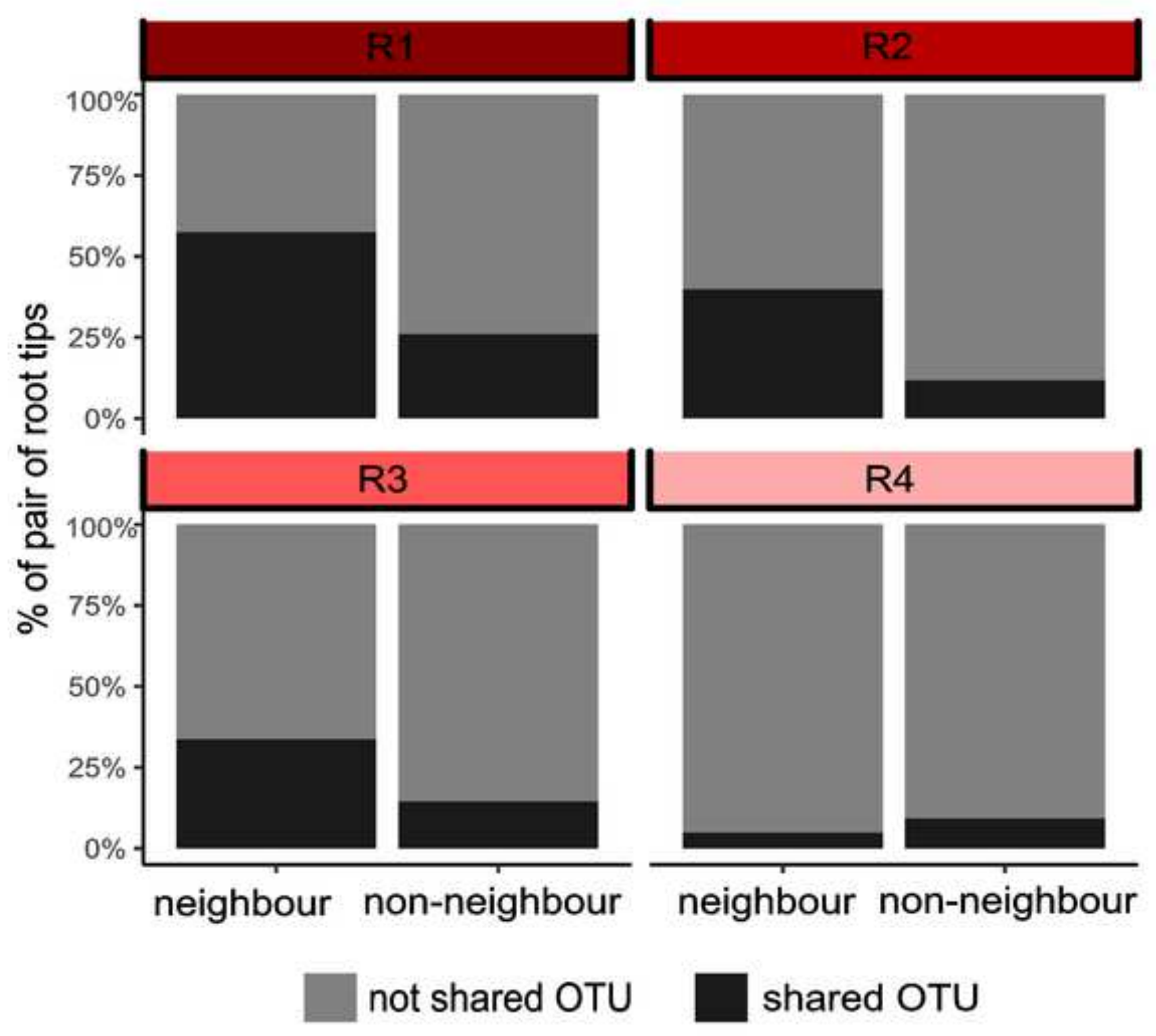




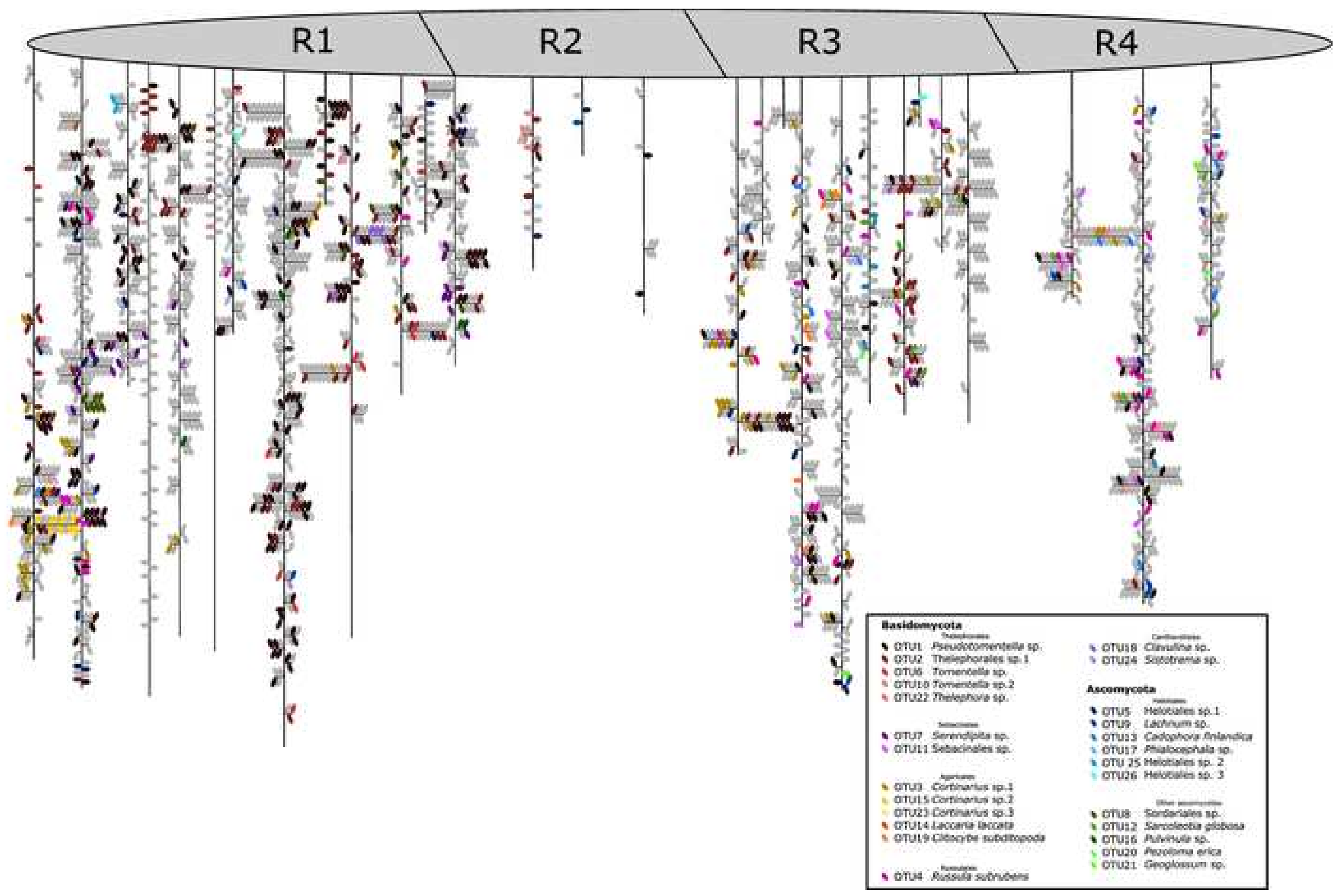


Table S1, S2, S3

Click here to access/download Electronic Supplementary Material Table_S1_S2_S3.docx 
Fig. S1 FigS1.tif

\section{Click here to access/download \\ Electronic Supplementary Material}


Fig. S2

FigS2.tif

\section{Click here to access/download \\ Electronic Supplementary Material}

